\title{
Failures in childbirth care
}

\author{
Brenda Ashcroft \\ School of Nursing and Midwifery, University of Salford, Salford
}

The study, first published in 2003, looks at the root causes of adverse events and near misses in obstetrics at seven hospital maternity units by interviewing 93 members of staff, identifying the areas of mismanagement in each case and thematically analysing them.

Journal of Health Services Research E Policy Vol 15 Suppl 1, 2010: 52-55 $\quad$ C The Royal Society of Medicine Press Ltd 2010

\section{Background}

Obstetric claims account for half of all NHS litigation bills with the cost of cerebral palsy cases being between $£ 1.5$ and $£ 8$ million.

\section{Aims of the study}

The aims of the project were to identify the underlying root causes of 37 cases of severe birth asphyxia in obstetrics likely to result in cerebral palsy claims, using a systems approach rather than a person approach.

\section{About the study}

The researcher studied 37 cases selected from seven maternity units within the north-west of England. The units studied covered a range of available facilities, including three large inner-city units with a high number of deliveries and significant obstetrical complications. Four of the seven units practised a team midwifery system in line with that recommended by the government in its Changing Childbirth guidance where most of the midwives there worked on a labour ward for a day at a time. Their experience, therefore was infrequent and patchy.

The other three units adopted the traditional approach of using block allocations to the labour ward which meant the midwives there had more consolidated experience. The researcher interviewed 93 members of staff altogether, of which 81 were midwives, two were consultants, 8 were registrars, one was a senior SHO (senior house officer) who had been acting in the role of registrar, and one was a junior SHO. A panel of expert witnesses was used to identify the areas of mismanagement in each case and these were thematically analysed.

\section{Practical findings}

For this work, the researcher used findings from an earlier study by the author to provide background information about latent failures within the system of care on the labour ward. In the 37 cases studied, all involved a child with severe birth asphyxia - where the child had problems receiving enough oxygen before, during or just after the birth process - and these children were taken to a neonatal unit. Many were considered to have a poor prognosis. Staff were interviewed to supplement information gathered from case records and recordings from cardiotocography (CTG) - the technical means of recording fetal heartbeat and contractions during childbirth.

An 'expert' assessment board then identified areas of mismanagement in each case where it was clear that different acts or omissions should have happened. This team included two consultant obstetricians, a consultant midwife, a midwifery risk manager, a consultant neonatologist, a neonatal midwife and the researcher. A root case analysis then took place, using accepted methods.

Of the 37 cases, 32 were adverse events and five were near misses, but all involved inappropriate action, a failure to take action or a delay in taking action. The researchers found that overall, at least 15 infants were likely to develop cerebral palsy due to a poor prognosis and the cases were often complicated. Less than half of the cases (16) were reported in trusts' incident reporting schemes, meaning 21 went unreported because either:

- there was a lack of recognition that an adverse event had just happened (some saw it as a near miss); or

- the case was not considered to be serious enough; or

- or in one case a midwife felt the consultant had acted inappropriately and contributed to the cause of the event, but did not want to openly criticize him. 
The largest amount of adverse events (20 out of 37) happened during a night shift.

\section{Type of failures}

Although human error was associated with every case looked at, this was linked to many factors such as a lack of clinical experience, lack of labour ward skills and knowledge, lack of training and lack of supervision for inexperienced members of staff.

In all cases, there were failures to identify obstetric complications and either a failure to act appropriately, a delay in doing so, or else inappropriate action was taken due to the above reasons. Various types of human failures happened including:

- not taking appropriate action at signs of fetal hypoxia (distress);

- the fetus not being monitored adequately;

- not recognizing an obstruction during labour;

- inappropriate use of Syntocinon - a drug given to help induce or continue labour;

- inappropriately failing to provide adequate supervision, support and guidance for junior and inexperienced staff;

- delays in providing adequate resuscitation for a newborn baby having breathing difficulties.

From the 37 cases, there were 13 emergency Caesarean sections, two deliveries by forceps, 10 by ventouse and 12 normal deliveries.

\section{System failures}

The researchers found there were 34 cases in which there were no guidelines or protocols to advise regarding clinical practice or organizational issues.

\section{Unsupported junior staff/inexperienced staff}

The seniority and experience of staff in these cases was also noted by the researchers as an issue. In more than half of the cases (19) the lead midwife providing care was either junior or inexperienced and many said they felt less confident in their labour ward skills.

In nine of the 15 cases of babies with the poorest prognosis, the main caregiver was a junior or inexperienced midwife. Similarly with medical staff, in 31 cases, problems arose due to the use of unsupervised junior medical staff who were in the first 'on-call' position for complications. In eight of the 15 cases involving infants with the poorest prognosis, the obstetricians involved were inexperienced. Problems also intensified when in 19 of the 37 cases, a paediatric registrar had to be 'crash called' quickly when the junior paediatrician attending lacked sufficient skill and experience to resuscitate the infants adequately.

\section{Communication and cultural issues}

Failures in communication were a problem in all the cases studied by the researchers, and were often related to culture on the labour ward - a system that is hierarchically-led and historically-based. When midwives and obstetricians had differences of opinion, the midwives often failed to seek assistance from the consultant because they feared criticism or that it would damage their working relationship with registrars.

There were failures to provide assistance to junior or inexperienced members of staff, and a failure for them to seek it when needed ( 29 cases). The culture of the labour ward seems to mean less experienced members of staff are expected to get on with the job and not seek help even when they want or need it because to do so would be considered as a lack of their ability or that they were unable to cope.

In 31 cases, problems arose when junior or inexperienced medical staff members were used in a 'first on-call' position for complications, also a cultural issue. In 19 cases it involving delayed infant resuscitation, the paediatric senior house officer (SHO) attended unsupervised, and the registrar had to be called in an emergency to assist. In 15 cases there was a difference of opinion between midwives and obstetricians regarding how and when to act, but no mechanism in place to support resolution.

\section{Heavy workloads and reduced midwifery staffing levels}

Workload was identified as an important factor and the researchers found that in 26 out of 37 cases, the adverse event or near miss happened during a very busy time on the labour ward when there were not enough midwives to manage cases safely.

In 10 of these 26 cases, there was a reduction of at least one midwife from the established number required on duty and in 10 other cases a poor skill-mix (too many junior midwives) put additional pressure on the already heavy workload.

The researchers found in 19 cases there was inappropriate use of high-risk procedures, involving the use of Syntocinon - a drug given to help induce or continue labour - and epidural blockades during busy times on the wards. There were also no guidelines or protocols to highlight the risks and special precautions with using Syntocinon.

In nine cases, there were high-risk or elective procedures (such as induction of labour and planned Caesarean sections) with inadequate midwifery staffing levels. All of these were evidence of latent errors in the 
system, said the researcher, creating an environment in which accidents were waiting to happen. There were also problems related to changeover of medical and midwifery staff in seven cases.

Seven cases involved midwives taking time away from clinical duties to perform other duties including clerical duties, domestic duties when other support was unavailable, and porter's duties, transferring clients to the wards following delivery.

\section{Training needs}

Training needs were apparent from interviewing the staff for the research. In more than half of cases (21), midwives and obstetricians lacked consistent updating in cardiotocography (CTG) interpretation, with midwives often lacking opportunities to do so because of workload, with no opportunities for time-out training.

In 18 cases, members of staff were unfamiliar with labour ward protocols and failed to follow them while in 11 cases, there were inadequate labour ward induction programmes for new members of staff. In 17 cases, paediatricians (SHOs) appeared to lack training in infant resuscitation.

In 13 cases staff lacked awareness of the signs of cephalopelvic disproportion (when the baby is too large to fit through the pelvis) and failed to identify the problem and take action.

\section{Lack of equipment and equipment failure}

Equipment problems also played a part and in 14 cases there was a lack of, or a failure of equipment, i.e. infant resuscitation apparatus and fetal blood sampling analysis machines.

\section{Key messages}

- Staff are reluctant to carry out an unplanned Caesarean section once labour has begun and take too long before deciding to intervene in this way;

- Not all adverse events are recorded in hospital trusts' critical incident reporting scheme but should be;

- There is a worrying lack of knowledge underpinning practice especially relating to the signs of obstruction during labour due to cephalopelvic disproportion (when the baby is too large to fit through the pelvis);

- Staff inexperience and lack of adequate supervision and guidance is involved in almost all cases of adverse events in childbirth;

- The culture of the labour ward means less experienced members of staff are expected to get on with the job and do not seek help even when they are unsure of what to do;

- Staff have become too casual about the use of Syntocinon (drug to help induce and continue labour) and underestimate the risks of using it;

- There is a need for training on a time-out basis for midwives and obstetricians so they can accurately interpret fetal heart recordings and practice emergency scenarios.
Only one of the labour wards in the maternity units studied had resuscitation apparatus in every room where deliveries might happen.

\section{Summary of main findings}

- Of 37 cases studied, at least 15 of the children had a poor prognosis and were likely to go on to develop cerebral palsy;

- More than half (56\%) of adverse event cases were not reported to the trust's critical incident reporting scheme;

- Three-quarters of cases of adverse events happened in intensely busy periods with inadequate numbers of midwives and most (20 out of 37) happened during night shifts;

- In almost all the cases (32) there were inexperienced midwives and obstetricians lacking labour ward skills, who were left unsupported and unsupervised with complicated cases;

- In three-quarters of cases, junior or inexperienced members of staff failed to seek help they needed because the culture of the labour ward discouraged them from doing so;

- In one-third of cases there was a failure to recognize cephalopelvic disproportion (when the baby is too large to fit through the pelvis) as an underlying cause of failure to progress in labour;

- In almost half of all cases there was an excessive or inappropriate use of Syntocinon;

- Inexperienced and unsupported clinicians who lack the necessary skills in infant resuscitation were often present at deliveries of babies with breathing difficulties at greatest risk of developing cerebral palsy.

\section{Conclusions, recommendations and implications for practice and policy}

- Midwives returning to work in the labour ward after a prolonged period need help and support from more experienced midwives because their knowledge, skills and confidence will have lessened while they were away;

- Mandatory multidisciplinary training for cardiotocography (CTG) interpretation and managing lifethreatening complications such as shoulder dystocia should be provided on a time-out basis;

- Consultants should be seen as a part of the labour ward team and not only as an advisor, so they need to maintain their clinical skills in this environment;

- The profile of cephalopelvic disproportion as an underlying cause of failure to progress in labour, Syntocinon, and labour complications should be raised among staff to increase their knowledge;

- Efforts should be made to change the existing hierarchical culture in the labour ward by encouraging 
more goodwill, cooperation and team work between different professional groups;

- Members of the medical team need to provide junior and more inexperienced practitioners with more supervision and support;

- Trusts need to set realistic minimum midwifery staffing levels for labour wards as this is a high-risk area prone to significant medical accidents and high financial litigation.

\section{Acknowledgements}

This briefing paper is based on work undertaken by Brenda Ashcroft, midwifery lecturer in the School of Nursing and Midwifery, University of Salford. We thank Adrian O'Dowd who helped compile the paper.

The full report, this research summary and details of other Patient Safety Research Portfolio work can be seen at http://www.pcpoh. bham.ac.uk/publichealth/psrp/commissioned.shtml

Conflict of interest: None. 